\title{
EMG Activation of the Vastus Medialis Oblique and Vastus Lateralis During Four Rehabilitative Exercises
}

\author{
Debra Kushion $^{1}$, Jessica Rheaume ${ }^{1}$, Kim Kopchitz ${ }^{1}$, Stephen Glass ${ }^{*}, 2$, Gordon Alderink ${ }^{1}$ and \\ Jann-Huei Jinn ${ }^{3}$ \\ ${ }^{1}$ Department of Physical Therapy, ${ }^{2}$ Department of Movement Science, ${ }^{3}$ Department of Statistics Grand Valley State \\ University, Allendale MI, USA
}

\begin{abstract}
The purpose of this study was to compare four common rehabilitation exercises used in physical therapy clinics for activating the vastus medialis oblique (VMO) and vastus lateralis (VL). Thirty-four subjects, aged 22-28 years, without patellofemoral pain syndrome (PFPS) (18 females and 16 males) were recruited. Subjects performed four exercises - straight leg raise with neutral and externally rotated hip positions (SLRN, SLRER), and short arc quad with neutral and externally rotated hip positions (SAQN, SAQER). The integrated electromyographic (iEMG) activity of the $\mathrm{VMO}$ and VL and were recorded, with means of the VMO, VL and VMO/VL compared across exercises. No significant difference was found for the $\mathrm{VMO} / \mathrm{VL}$ ratio across the four exercises $(\mathrm{p}=0.147)$. However, for the VMO and VL individually, a significant difference was found $(\mathrm{p}=0.0001)$, SAQER was significantly greater than SLRN and SLRER; SAQN was significantly greater than SLRN and SLRER.

These findings suggest that exercises including short arc quad knee extensions are more effective for both the VMO and VL activation than those incorporating straight leg raises, and potentially more beneficial in the rehabilitation of patients with PFPS.
\end{abstract}

Keywords: Knee pain, patellofemoral pain, knee rehabilitation, vastus medialis, VMO.

\section{INTRODUCTION}

Patellofemoral pain syndrome (PFPS) has been defined, in the absence of other pathology, as anterior or retropatellar pain $[1,2]$, and has been reported to be the primary cause of knee pain in the United States [3, 4]. It is a common problem that affects athletic and non-athletic people [5], most commonly women between the ages of 18-40. In the general population the incidence of PFPS has been reported as high as one in four individuals [3, 6, 7]. Patients with PFPS present with many symptoms including diffuse pain, patellofemoral joint crepitus, peripatellar swelling, and buckling or locking of the knee $[1-3,5]$. This syndrome is typically aggravated by activities such as prolonged sitting, climbing stairs, squatting, and kneeling $[1,2,6]$. Because weakness of the vastus medialis oblique muscle (VMO) is hypothesized to be linked to PFPS, treatment often involves strengthening the VMO, which serves to correct lateral tracking of the patella.

A muscular imbalance between the VMO and the vastus lateralis (VL), and improper timing of activation between the two muscles are perceived to result in abnormal patellar tracking and to be the two most common causes of PFPS [1, $6,8]$. The electrical activation of the VMO and VL are often expressed as a ratio value, (VMO/VL). Significantly increased $\mathrm{VMO} / \mathrm{VL}$ values indicate preferential VMO activation [5, 9-11]. Many clinicians focus their treatment of PFPS

*Address correspondence to this author at the College of Interdisciplinary Studies, Department of Movement Science, Grand Valley State University, 1 Campus Dr. Allendale, MI 49401, USA; Tel: 616-331-8126; Fax: 616331-8658; E-mail: glassst@gvsu.edu on restoring the strength of the $\mathrm{VMO}$ in order to increase the $\mathrm{VMO} / \mathrm{VL}$ ratio, and also on improving the timing of the VMO and VL contractions in order for them to occur simultaneously. There are several studies that support these theories as a treatment for PFPS. However, there have also been inconsistent results found by various research studies concerning the timing and $\mathrm{VMO} / \mathrm{VL}$ ratio. In a literature review performed by Westfall and Worrell, 15 years of research on the VMO muscle's role in knee rehabilitation was evaluated. They found that none of the rehabilitation methods consistently resulted in preferential VMO activation during various lower extremity exercises [12]. A number of studies have examined the $\mathrm{VMO} / \mathrm{VL}$ ratio during open chain knee extension and have found that both the VMO and VL are active throughout the full range of motion $[5,3,13,14]$. Several researchers have investigated the effect of specific knee angle on the activation of the VMO and reported conflicting results on which position is most effective. The arguments are between a mid-range knee flexion (90 to 30 degrees) versus end-range extension position (30 to 0 degrees) $[15,16]$. Using a dynamic knee extension exercise would allow for activation of the VMO throughout the range of motion, which would satisfy both positions mentioned previously.

Testing subjects with and without PFPS, five isometric exercises were examined by Laprade et al. [3]. Only one of the five exercises used was found to be effective for preferential VMO recruitment (medial tibial rotation with $110^{\circ}$ of knee extension); however, this is not a commonly used exercise in the clinical setting. For this reason, the authors recommended a study be conducted with more commonly used exercises. They also found no difference in 
muscle recruitment between subjects with and without PFPS. Thus, rehabilitation techniques to strengthen the VMO can be examined using subjects without PFPS. This finding is supported by Willis et al. [11], who found no difference between symptomatic (PFPS) subjects and the control group. The researchers specifically looked at the effect of openstance cycling protocol (OSCP) versus traditional cycling foot position (TCFP) for preferential VMO muscle activation, as measured by surface EMG. The results showed significantly higher ratio values during all readings for the OSCP group compared to the TCFP group. This indicated that an externally rotated lower extremity position induced greater VMO recruitment in both subject populations.

Livecchi et al. [17], investigated the $\mathrm{VMO} / \mathrm{VL}$ value during straight leg raise (SLR) and knee extension (KE) with the lower extremity in neutral and externally rotated (ER) positions. No differences in $\mathrm{VMO} / \mathrm{VL}$ ratio were found between the four exercises; however, the authors only used $5 \%$ of body weight on the subjects' ankle, and studied only males. Roush et al. [18] conducted a study in support of SLR with ER for rehabilitation of anterior knee pain, finding improved patient outcomes and cost effectiveness. Similarly, Sykes and Wong [19], concluded that SLR with ER was advantageous, especially with the addition of an ankle weight.

Research has indicated that pain caused by patellofemoral pain syndrome (PFPS) can be significantly reduced or eliminated using physical therapy $[1,2,18]$. However, based on a review of literature, there is still discrepancy as to which exercises best target the vastus medialis oblique (VMO) in comparison to the vastus lateralis (VL). Several variations to exercises have been studied, such as the effect of hip or tibial positioning [3,20], but as of yet no criterion method has been determined. Continued research should be done to further investigate commonly used quadriceps exercises in an effort to reach a higher standard of care for patients with PFPS.

The purpose of this study was to investigate the motor unit recruitment of the vastus medialis oblique (VMO) muscle during four clinically popular strengthening exercises that are thought to preferentially activate the VMO over the vastus lateralis (VL) muscle. The exercises were chosen based on responses received from a questionnaire given to local physical therapy clinics and past research. By investigating the motor unit recruitment of the VMO during these exercises, we hoped to determine which one, if any, is able to enhance preferential activation of the VMO for strengthening of the VMO and possible treatment of PFPS.

\section{METHODS}

This study was a repeated measures design. Surface EMG was used to analyze the motor recruitment of the vastus medialis oblique and vastus lateralis muscles during four different leg exercises. Approval for this study was obtained from the University Human Subjects Review Board. Subjects were found using a convenience sample of volunteers from the University population, and its surrounding areas. Thirty-four subjects were recruited to participate in this study (16 male and 18 female). Informed consent was obtained from each participant prior to any testing. Subjects were given a health history questionnaire, which also served to confirm that the inclusion/exclusion criteria necessary for admission to the study were met. One female subject was excluded because she did not meet the body fat inclusion criteria (criteria: Men $\leq 25 \%$, women $\leq$ $31 \%$ ). The remaining 33 subjects were eligible to participate. Subjects were assigned numbers 1-33 to guarantee their confidentiality. All testing was performed in the University Human Performance Laboratory.

Measurements of subjects' height in centimeters, weight in kilograms, and body fat percentage were recorded on the day of testing. Body density was estimated by using a skin fold caliper and three-site equation [21], with body fat calculated using the Siri equation [22]. Body fat percentage was limited to insure adequate EMG signal through subcutaneous tissues. Subjects were given a brief explanation of the EMG equipment along with a description and demonstration of all four exercises. Subject characteristics are shown in Table $\mathbf{1}$.

\section{Equipment and Instruments}

The Biopac ${ }^{\circledR}$ Tel-100 EMG system [23] (Biopac Systems, Inc. 2007, Goleta, CA) was used to measure muscle electrical activity and record the data from each subject. The EMG data were analyzed using Acqknowledge $^{\mathrm{TM}}$ software. Bipolar adhesive surface electrodes (Ag$\mathrm{AgCl}$ ) were used over the muscle bellies of the VMO and VL. Skin impedance was measured using an ohmmeter, and was held below $10,000 \mathrm{ohms}$ to maximize signal strength [24].

\section{Procedures}

Subjects were recruited through email and fliers posted on the University campuses. A counterbalance design for the order in which exercises were performed was used to eliminate any test order effects. After the preliminary procedures of collecting height, weight, and body fat, each subject was prepped for electrode placement. The dominant leg, as determined by handedness of the subject, was used for all subjects for electrode placement. The skin was prepped for electrode placement by shaving the designated areas to remove hair and dead skin cells. Then, the areas were abraded using a coarse pad and rubbed clean with rubbing alcohol and a towel. These procedures were

Table 1. Subject Characteristics (Mean \pm SD)

\begin{tabular}{|c|c|c|c|c|}
\hline Group & Age (y) & Height (m) & Mass (kg) & Percent Body Fat \\
\hline \hline Males & $24.90 \pm 1.48$ & $1.81 \pm 0.07$ & $82.90 \pm 14.18$ & $10.80 \% \pm 3.90$ \\
\hline Females & $23.80 \pm 1.25$ & $1.68 \pm 0.07$ & $64.60 \pm 6.82$ & $21.10 \% \pm 4.70$ \\
\hline Combined & $24.30 \pm 1.47$ & $1.74 \pm 0.09$ & $73.50 \pm 14.26$ & $16.10 \% \pm 6.75$ \\
\hline
\end{tabular}


followed until the skin impedance was found to be less than 10,000 ohms using a standard ohmmeter. The electrodes were placed parallel to the direction of the muscle fibers on the vastus medialis oblique (VMO) and vastus lateralis (VL). The fibers of the VMO run at approximately a 55-degree angle medial to the quadriceps tendon, and the electrode was placed $20 \%$ of the distance from the medial joint line of the knee to the anterior superior iliac spine [25]. The fibers of the $\mathrm{VL}$ are at 12 to 15 -degrees lateral to the quadriceps tendon; the electrode was placed at the midpoint between the head of the greater trochanter and the lateral femoral epicondyle [25]. Ground electrodes were placed on the patella and 6 to 8 $\mathrm{cm}$ from the inferior pole of the patella along the bony shaft of the anterior tibia.

In order to compute normalized data for $\mathrm{VMO} / \mathrm{VL}$ ratios the subject was seated at the end of a treatment table to perform a maximal voluntary isometric contraction (MVIC) of the quadriceps against manual resistance, described by Kendall [26], while the EMG activity of the VMO and VL was recorded. The subject performed one repetition of the knee extension as a "make-test" for five seconds [26]. However, we found that the MVIC data were quite variable, with unreliable measures through multiple trials as well as within trial. Therefore in order to normalize the dynamic movements of the experimental tests to a dynamic maximum, the researchers chose to normalize each muscle the data to the highest recorded IEMG value for that specific muscle. This assured that consistency for normalization would exist within each muscle, and be based on a dynamic rather than static movement. Kellis and Baltzopoulos [27] found that dynamic methods of normalization produced significantly greater EMG signals than static methods, and concluded that a dynamic method of normalization is more appropriate when performing dynamic testing.

After being repositioned, each subject was given a brief description and demonstration of the exercises, and given time to practice before beginning data collection. Each subject was then fitted with a five pound, sand-filled weight that was placed over the superior portion of the ankle and held in place with athletic tape to provide some resistance without causing fatigue during the trials. In counterbalanced order, the exercises were carried out as follows: During the straight leg raise with neutral foot position (SLRN), the subject was supine with the non-dominant knee bent to $90^{\circ}$, as measured by a goniometer, and their foot flat on the treatment table. The foot of the dominant leg was set to neutral, $0^{\circ}$, and the leg remained straight. A straight edge was held perpendicularly across the non-dominant leg to act as a guide for range of motion (Fig. 1). With the metronome set at 40 beats/minute, the subject was given the verbal cue to, "lift, lower, rest," at each beat. The subject was stopped after thirty seconds of data were recorded. The same exercise was repeated with the dominant leg in $45^{\circ}$ of external rotation, as measured through the foot (SLRER).

The last two exercises were short arc quadriceps extension with a neutral (SAQN) and externally rotated foot position (SAQER). For the SAQ exercises, the subject was supine with a bolster under their dominant leg to maintain a knee angle of $60^{\circ}$, and ankle over the edge of the treatment table, in order to maintain either neutral or externally rotated leg position (Fig. 2). The verbal cues were "lift" at the first beat, and "lower" at the second beat. The metronome was changed to 30 beats/minute for both SAQ exercises to allow for a more comfortable cadence.

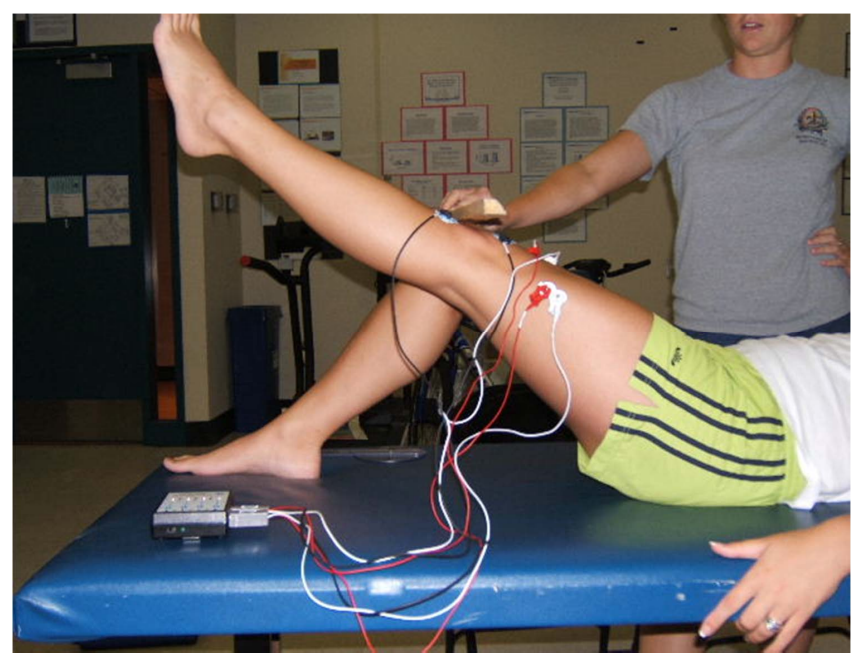

Fig. (1). Straight Leg Raise Positioning. Image shows neutral foot position (SLRN). SLRER (straight leg external rotation) consisted of a 45 degree external rotation. VMO, VL and ground electrodes shown. Ankle weight not shown.

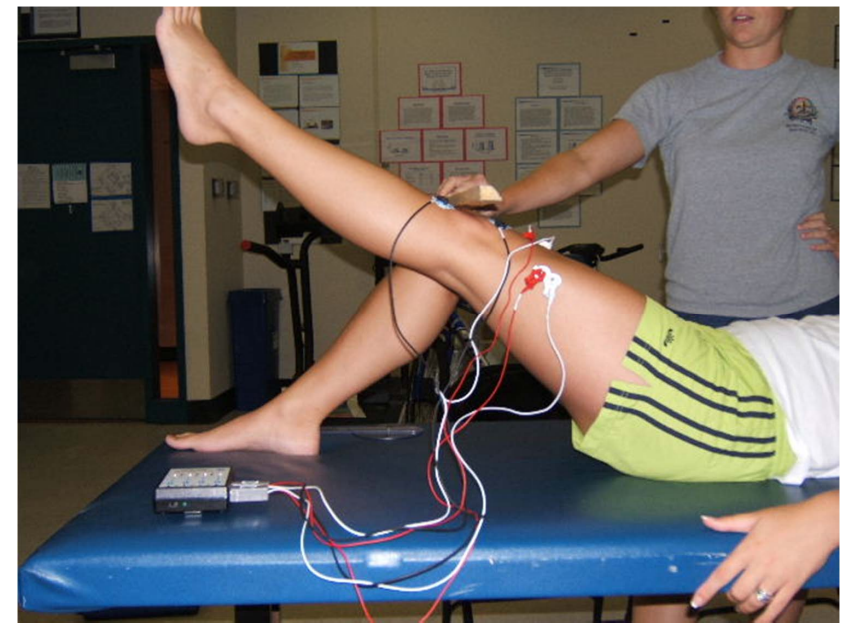

Fig. (2). Short Arc Quadriceps Position (shown with Neutral foot (SAQN), in extension). SAQER (short arc quadriceps external rotation) had 45 degree external rotation. Bolster under knee ensures 60 degree angle at neutral position. VMO, VL and ground electrodes shown. Ankle weight not shown.

During each repetition of all the exercises, a designated researcher marked the concentric and eccentric phases using the computer keyboard to mark phase transition. Subjects were allowed a three-minute recovery period in between each exercise. The EMG data were sampled at $1,000 \mathrm{~Hz}$ and amplified 2,000 times by the Tel-100 unit.

\section{Data Analysis}

Raw EMG data were collected and then filtered to reduce the amount of artifact. A high pass filter (Blackman $-67 \mathrm{~dB}$ ) was used with a cutoff frequency of $4 \mathrm{~Hz}$ and 1000 coefficients. Data were rectified and integrated, and standardized to account for variations in lift speed and number of samples. An average of the middle five repetitions was calculated for each exercise using only the concentric 
portion of the contraction in order to improve reliability [28, $29,30]$. Data analysis was performed using Statistical Package for the Social Sciences (SPSS) software version 12.0. The data were analyzed using a one-way analysis of variance (ANOVA) with an alpha level of 0.05 . If significance was found using the one-way ANOVA, a Bonferonni post-hoc analysis was performed. In order to protect against type I error, the significance levels for post-hoc analyses were adjusted to 0.008 using Bonferroni's correction (adjusted $\alpha=$ $\alpha / \#$ of comparisons $=0.05 / 6=0.008$ ). The ANOVA and post-hoc were performed to analyze the data separately for each muscle, as well as for the $\mathrm{VMO} / \mathrm{VL}$ ratio. By using a one-way repeated measures design it can be assured that differences across conditions are a function of the exercises, not individual physiologic differences [31].

\section{RESULTS}

Three, one-way repeated measures ANOVAs were performed to compare the integrated EMG means across the four exercises for the vastus medialis oblique and vastus lateralis muscles separately, as well as the $\mathrm{VMO} / \mathrm{VL}$ ratio $(\alpha$ $=0.05$ ). The comparisons for the $\mathrm{VMO} / \mathrm{VL}$ ratio across the four exercises are shown in Fig. (3). A one-way ANOVA was performed for the $\mathrm{VMO} / \mathrm{VL}$ ratio, which indicated no significant differences across the exercises $(p=0.147)$. Fig. (4) displays the comparison of the VMO alone across the four exercises. Results of a one-way ANOVA indicated significant differences were present across the exercises $(\mathrm{p}=0.0001)$.

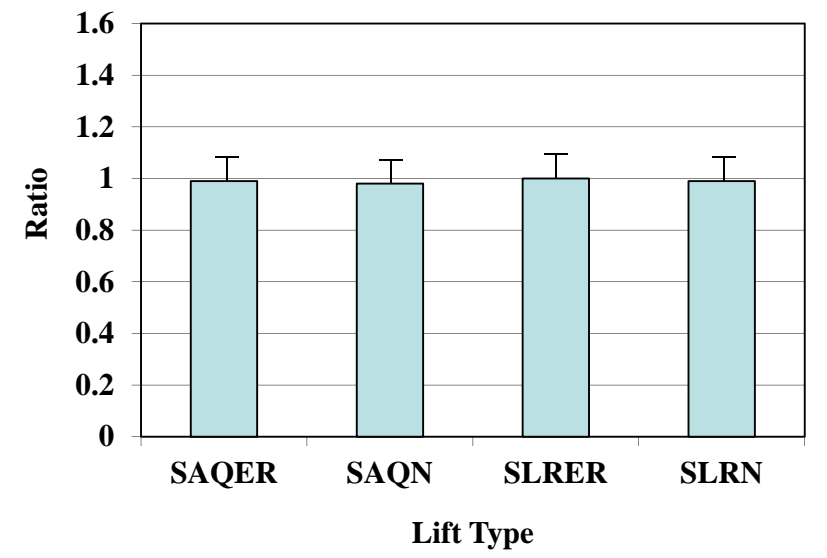

Fig. (3). VMO/VL Ratio across movements. No significant differences seen in $\mathrm{VMO} / \mathrm{VL}$ across SAQER (short arc quad external rotation), SAQN (short arc quad neutral), SLRER (straight leg raise external rotation) and SLRN (straight leg raise neutral foot).

The post-hoc analysis for the VMO indicated the following significant differences: short arc quad with external rotation $(\mathrm{SAQER}=0.046 \pm 0.029 \mathrm{v} * \mathrm{~s})$ was significantly greater than straight leg raise with neutral leg $\left(\mathrm{SLRN}=0.019 \pm 0.010 \mathrm{v}^{*} \mathrm{~s}\right)$ and straight leg raise with external rotation $\left(\bar{S} L R E R=0.021 \pm 0.009 \mathrm{v}^{*} \mathrm{~s}\right)(\mathrm{p}=0.0001)$, and short arc quad with neutral leg $(\mathrm{SAQN}=0.046+0.023$ $\left.\mathrm{V}^{*} \mathrm{~s}\right)$ was also significantly greater than SLRN $(\mathrm{p}=0.0001)$ and SLRER $(p=0.0001)$. No significant differences were seen for the VMO between SAQN and SAQER or between SLRER and SLRN. Comparison across the four exercises for the VL alone is displayed in Fig. (5).

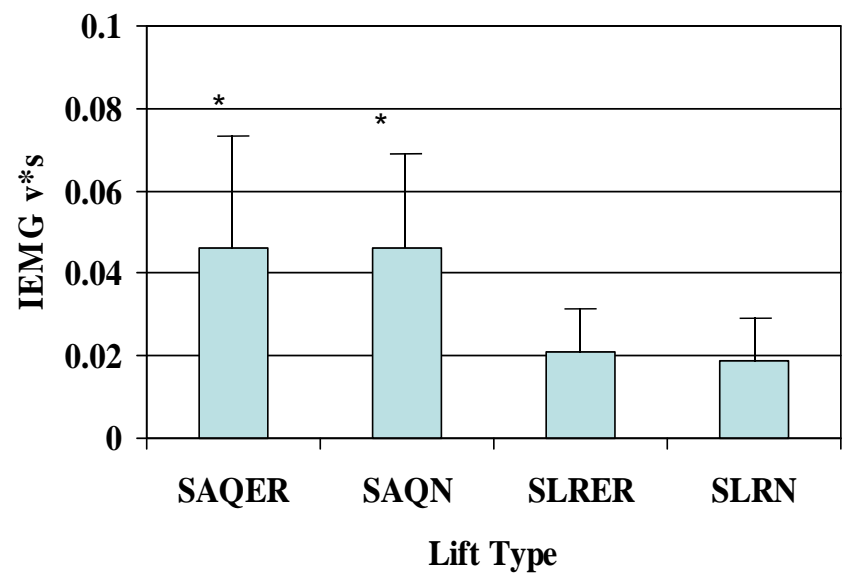

Fig. (4). Muscle activation of VMO across movements. SAQER (short arc quad external rotation) and SAQN (short arc quad neutral foot) elicited significantly greater activation than straight leg raises in either external rotation (SLRER) or neutral foot (SLRN).

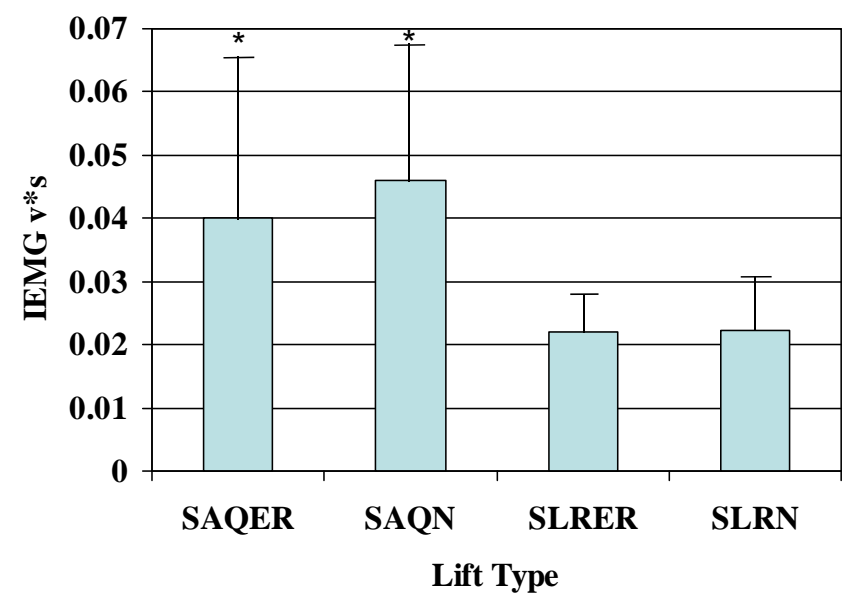

Fig. (5). Muscle Activation of VL across movements. SAQER (short arc quad external rotation) and SAQN (short arc quad neutral foot) showed significantly greater activation than straight leg raises in either external rotation (SLRER) or neutral foot (SLRN).

Results from the one-way ANOVA $(p=0.0001)$ and Bonferonni post hoc analysis indicated SAQER VL activation $\left(0.40 \pm 0.026 \mathrm{v}^{*} \mathrm{~s}\right)$ was significantly greater than $\operatorname{SLRN}(0.022 \pm 0.009 \mathrm{~V} * \mathrm{~s})$ and SLRER $(0.022 \pm 0.009$ $\mathrm{p}=0.001)$. SAQN VL activation $(0.046 \pm 0.022)$ was also significantly greater than SLRN $(\mathrm{p}=0.0001)$ and SLRER $(p=0.0001)$. No significant differences were found between SAQN and SAQER $(p=1.000)$ or between SLRER and SLRN ( $p=1.000)$ for activation of the vastus lateralis.

\section{DISCUSSION}

The primary focus of this study was to compare the muscular activation of the vastus medialis oblique (VMO), vastus lateralis (VL), and the $\mathrm{VMO} / \mathrm{VL}$ ratio across four common rehabilitation exercises: straight leg raise with neutral (SLRN) and externally rotated (SLRER) hip positions, and short arc quads with neutral (SAQN) and externally rotated (SAQER) hip positions. The VMO/VL ratios were all very similar, ranging from 0.99 to 1.0 . Because there was no significant difference between the ratios across the four exercises, we cannot conclude based on this number alone that one of these exercises is better than 
the others for preferentially recruiting the VMO over the VL. All of the exercises did have a ratio close to 1.00 , which would indicate equal activation of the two muscles. Based on these values, we can suggest that all four exercises can lead to fairly equal activation the VMO and VL, possibly allowing for simultaneous strengthening during rehabilitation. Even though the VMO is not being activated more than the VL, it is also important to note that the VL is also not being activated over the VMO. This is significant because increasing the strength of the VL could further increase a lateral force vector on the patella, which often contributes to PFPS. Therefore, rehabilitation exercises that have increased $\mathrm{VMO} / \mathrm{VL}$ ratios are needed to effectively strengthen the VMO and correct the muscular imbalance [3, $5,11]$.

Although the VMO/VL ratios were not different among the exercises, there were differences in VMO and VL activation when analyzed individually. The short arc quad (SAQ) exercises were found to generate significantly more overall activation of the VMO and VL muscles than the straight leg raise (SLR) exercises. The anatomy and kinesiology of the muscles provide possible explanations as to why the SAQ exercises activate the quadriceps to a greater extent. The VMO and VL assist with knee extension, which could explain why there is an increase in EMG activity with SAQ due to the terminal knee extension component. The rectus femoris $(\mathrm{RF})$ overlaps the $\mathrm{VMO}$ and $\mathrm{VL}$, and plays a role in hip flexion. Because of increased activation of the RF during hip flexion exercises, it has been hypothesized that SLR exercises would show less EMG activity for the VL and VMO [17, 32]. The type of muscle contractions that occur during the exercises may also effect the overall activation of the VMO and VL. The SAQ exercise involves dynamic knee extension involving concentric and eccentric activation of the quadriceps muscles, leading to greater recruitment of the VMO and VL. A SLR requires the VMO and VL to stabilize the knee in extension, which closely resembles an isometric contraction during flexion and extension of the hip. This type of contraction may require less force from the VMO and VL, and therefore result in a lower activation. Another possible explanation is that as the knee nears extension, the moment arm of the quadriceps decreases and therefore more muscle tension is needed to perform the movement [33].

While the two main exercise categories were found to have differences, the externally rotated hip position did not result in a significantly different amount of activation when compared to the neutral leg position within each exercise. These findings are similar to earlier studies, which reported no significant changes in EMG activity of the VMO and VL when the hip position was changed [8, 17, 20,34]. Other researchers have hypothesized that there will be an increase in VMO activity with external rotation of the hip because of the VMO's muscular fibers originating from the adductor magnus muscle. Based on this thinking, when the hip is placed in an externally rotated position and flexed using the adductors, the VMO would be stimulated, therefore causing an increase in EMG activity [10, 17-19]. This study found, however, that there was no significant difference between VMO activation during lateral rotation or neutral positioning of the hip. These findings agree with other studies that have suggested that it is not possible to preferentially activate the VMO. The femoral nerve innervates the rectus group,

including the VMO and VL, therefore making it unlikely to activate one muscle more so than the others [17, 34, 35].

Many studies have suggested that women are more likely to fatigue with knee extensor exercises than men, and are also at an increased risk for PFPS because of anatomical and kinesiological differences, such as an increased Q-angle. Despite PFPS being more prevalent in females [17, 19, 3638], several studies reviewed used exclusively male subjects $[39,40]$. In order to broaden the patient population that this study is applicable to, we chose to include both males and females. Differences between the two genders were not analyzed in this study. Instead, the data were combined to account for both males and females, allowing the results to be generalized to both populations.

While this study had a sample containing a nearly equal number of males and females, there were still some limitations to the subject population. A convenience sample was used for subject recruitment, which yielded a large majority of subjects in their mid-twenties, thus limiting the ability to generalize the results to a wide age range. Another limitation to the subject population was the body fat percentage exclusion criteria. In order to decrease interference of EMG signals, the subjects were required to have an acceptable amount of body fat [21, 22], which excluded overweight individuals from participating in the study. This limits the anthropometric characteristics of subjects, and therefore the results may not be representative of the general public. Some researchers have reported PFPS to be more common with an athletic population [8,37], such as our subjects, while others state it is also common in the general population $[2,5]$.

Along with being applicable to a wide population, the researchers wanted to study exercises that can be easily used in the clinical setting. The four chosen exercises are used frequently in physical therapy clinics and have been investigated in previous studies with conflicting results [16, 17, 19, 41]. All of the exercises tested produced relatively high VMO/VL ratios, however this study indicates that there is a statistically significant increase in the EMG activity of the VMO and VL during the SAQ exercises over the SLR, regardless of hip position.

While these exercises are typically used to treat patients with PFPS, the current study did not include subjects with PFPS. Previous research has shown no significant difference between subjects with and without PFPS when comparing $\mathrm{VMO} / \mathrm{VL}$ ratios across exercises [3, 5, 11, 36, 42].

To decrease limiting factors identified in previous research, the current study design attempted to incorporate several suggested ideas from previous authors. Earlier studies have suggested that more weight than $5 \%$ of lean body mass was needed to increase muscle recruitment [17], however Sykes and Wong found that weight less than 5\% of lean body mass (specifically, 2.5 pounds) produced a $7.5 \%$ increase in VMO activity with an externally rotated hip position [19]. Similarly, results from the present study indicate that a 5-pound ankle weight, along with lower extremity weight and gravity, was sufficient for distinguishing differences in VMO and VL activation. This weight is easy for patients to overcome, practical for therapists to prescribe for patients, and still provides the resistance needed for proper muscle facilitation. Finding 
both a weight and exercise that are realistic for clinical use is very important. Performing exercises with an externally rotated leg position is frequently used in research; however, many of the subjects in this study had difficulty maintaining the lower extremity external rotation at a constant angle of 45 degrees. Some subjects tended to turn their leg back to a neutral position, rather than externally rotated, which may have had an effect on the results. Verbal reminders were needed to help the subjects maintain the proper lower extremity position, which may also be required of clinicians during rehabilitation, if they choose to use this position.

Knutson et al. [29] found that using same-day recordings reduced variability in results without moving or replacing electrode pads. Based on this, all recordings for this study were performed in a single time period for each subject. With any surface EMG study, crosstalk between electrodes is also a concern. Bipolar placement of electrodes, similar to the ones used in this study, has been suggested to have minimal crosstalk interference $[19,25,43]$.

Recommendations for future research include considering the effect of Q-angle on recruitment patterns of the VMO and VL, as well as a longitudinal study including these exercises in the rehabilitation protocol in a more general patient population including a wider range of ages, body types, and activity levels.

\section{CONCLUSION}

Although there was no significant difference in VMO/VL ratios across the four exercises, the short arc quad exercises resulted in significantly greater muscle recruitment for the VMO and VL muscles independently. This finding suggests that exercises including short arc quad knee extensions are more facilitative than those incorporating straight leg raises for activating the VMO and VL muscles.

\section{ACKNOWLEDGEMENT}

Declared none.

\section{CONFLICT OF INTEREST}

Declared none.

\section{REFERENCES}

[1] Cowan S, Bennell KL, Crossley KM, Hodges PW, McConnell J. Simultaneous feed forward recruitment of the vasti in untrained postural tasks can be restored by physical therapy. J Orthop Res 2003; 21(3): 553-8.

[2] Cowan S, Bennell KL, Crossley KM, Hodges PW, McConnell J. Physical therapy alters recruitment of the vasti in patellofemoral pain syndrome. Med Sci Sports Exerc 2002; 34(11): 1879-85.

[3] Laprade J, Culham E, Brouwer B. Comparison of five isometric exercises in the recruitment of the vastus medialis oblique in persons with and without patellofemoral pain syndrome. J Orthop Sports Phys Ther 1998; 27(3): 197-204.

[4] Malek MM, Mangine RE. Patellofemoral pain syndromes: A comprehensive and conservative approach. J Orthop Sports Phys Ther 1981; 2(3): 108-16.

[5] Sheehy P, Burdett RG, Irrgang JJ, VanSwearingen J. An electromyographic study of vastus medialis oblique and vastus lateralis activity while ascending and descending steps. J Orthop Sports Phys Ther 1998; 27(6): 423-9.

[6] Hung YJ, Gross MT. Effect of foot position on electromyographic activity of the vastus medialis oblique and vastus lateralis during lower-extremity weight-bearing activities. J Orthop Sports Phys Ther 1999; 29(2): 93-105.
[7] Zappala FG, Taffel CB, Scuderi GR. Rehabilitation of patellofemoral joint disorders. Orthop Clin North Am 1992; 23(4): 555-66.

[8] Miller JP, Sedory D, Croce RV. Leg rotation and vastus medialis oblique/vastus lateralis electromyogram activity ratio during closed chain kinetic exercises prescribed for patellofemoral pain. J Athl Train 1997; 32(3): 216-20.

[9] Hungerford DS \& Barry M. Biomechanics of the patellofemoral joint. Clin Orthop 1979; 144 (1): 9-15.

[10] Thiranagama R. Nerve supply of the human vastus medialis muscle. J Anat 1990; 170: 193-8.

[11] Willis FB, Burkhardt EJ, Walker JE, Johnson MA, Spears TD. Preferential vastus medialis oblique activation achieved as a treatment for knee disorders. J Strength Cond Res 2005; 19(2): 286-91.

[12] Westfall DC, Worrell TW. Anterior knee pain syndrome: Role of the vastus medialis oblique. J Sports Rehabil 1992; 1: 317-25.

[13] Salzman A, Torburn L, Perry J. Contribution of rectus femoris and vasti to knee extension. Clin Orthop 1993; 290: 236-43.

[14] Souza DR, Gross MT. Comparison of vastus medialis oblique: Vastus lateralis muscle integrated electromyographic ratios between healthy subjects and patients with patellofemoral pain. Phys Ther 1991; 71(4): 310-20.

[15] Matheson JW, Kernozek TW, Fater DCW, Davies GJ. Electromyographic activity and applied load during seated quadriceps exercises. Med Sci Sports Exerc 2001; 33(10): 1712-25.

[16] Sczepanski TL, Gross MT, Duncan PW, Chandler JM. Effect of contraction type, angular velocity, and arc of motion on VMO:VL EMG ratio. J Orthop Sports Phys Ther 1991; 14 (6): 256-62.

[17] Livecchi NM, Armstrong CW, Cordova ML, Merrick MA, Rankin $\mathrm{JM}$. Vastus lateralis and vastus medialis obliquus activity during a straight-leg raise and knee extension with lateral hip rotation. J Sport Rehab 2002; 11(2): 120-6

[18] Roush MB, Sevier TL, Wilson JK, et al. Anterior knee pain: A clinical comparison of rehabilitation methods. Clin J Sport Med 2000; 10 (1): 22-8.

[19] Sykes K, Wong YM. Electrical activation of vastus medialis oblique muscle in straight leg raise exercise with different angles of hip rotation. Physiotherapy 2003; 89 (7): 423-30.

[20] Willett GM, Paladino JB, Barr KM, Korta JN, Karst GM. Medial and lateral quadriceps muscle activity during weight-bearing knee extension exercise. J Sport Rehab 1998; 7 (4): 248-57.

[21] Pollock ML, Schmidt DH, Jackson AS. Measurement of cardiorespiratory fitness and body composition in the clinical Setting. Compr Ther 1980; 6(9): 12-27.

[22] Siri WE. Body composition from fluid spaces and density: Analysis of methods in techniques for measuring body composition. Washington DC: Natl Acad Science, Natl Res Council 1961; pp. 223-44

[23] Biopac Systems, Inc. MP Systems Hardware Guide. Available at: http://www.biopac.com/ManualDetails.asp?ID=12, [cited: Jan 2007].

[24] Basmajian JV. Muscles alive: their functions revealed by electromyography. $5^{\text {th }}$ ed. Baltimore, MD: Williams \& Wilkins 1985.

[25] Zipp P. Recommendations for the standardization of the lead position in surface electromyography. Eur J Appl Physiol 1982; 50 (1): 41-54.

[26] Kendall FP. Muscles: Testing and function, with posture and pain: Fourth ed. Philidelphia, PA: Lippincott Williams and Wilkins 1993.

[27] Kellis E, Baltzopoulos V. The effects of normalization method on antagonistic activity patterns during eccentric and concentric isokinetic knee extension and flexion. $\mathrm{J}$ Electromyogr Kinesiol 1996; 6 (4): 235-45.

[28] Donaldson S, Donaldson M. Multi-channel EMG assessment and treatment techniques. In: Cram JR, Ed. Clinical EMG for Surface Recordings. Nevada City, CA: Clinical Resources 1990; vol. 2.

[29] Knutson LM, Soderburg GL, Ballantyne BT, Clarke WR. A study of various normalization procedures for within day electromyographic data. J Electromyogr Kinesiol 1994; 4(1): 4759.

[30] Mathur S, Eng JJ, MacIntyre DL. Reliability of surface EMG during sustained contractions of the quadriceps. J Electromyogr Kinesiol 2005; 15 (1): 102-110. 
[31] Portney LG, Watkins MP. Foundations for clinical research: applications to Practice. Upper Saddle River NJ: Prentice Hall Inc. 2000; pp. 127-33.

[32] Soderberg GL, Cook TM. An electromyographic analysis of quadriceps femoris muscle setting and straight leg raising. Phys Ther 1983; 63(9): 1434-8

[33] Neumann D. Kinesiology of the musculoskeletal system: foundations for physical rehabilitation. St. Louis, MO: Mosby Inc 2002.

[34] Davlin CD, Holcomb WR, Guadagnoli MA, Worrell TW. The effect of hip position and electromyographic biofeedback training on the vastus medialis oblique: vastus lateralis ratio. J Athl Train 1999; 34(4): 342-50.

[35] Stensdotter AK, Hodges PW, Mellor R, Sundelin G, Hager-Ross C. Quadriceps activation in closed and in open kinetic chain exercise. Med Sci Sports Exerc 2003; 35(12): 2043-7.

[36] Boucher JP, King MA, Lefebvre R, Pepin A. Quadriceps femoris muscle activity in patellofemoral pain syndrome. Am J sports Med 1992; 20(5): 527-32

[37] Coqueiro KRR, Bevilaqua GD, Berzin F, Soares AB, Candolo C, Monteiro PV. Analysis on the activation of the VMO and VLL muscles during semisquat exercises with and without hip adduction in individuals with patellofemoral pain syndrome. J Electromyogr Kinesiol 2005; 15 (6): 596-603.

[38] Pincivero DM, Gandhi V, Timmons MK, Coelho AJ. Quadriceps femoris electromyogram during concentric, isometric and eccentric phases of fatiguing dynamic knee extensions. J Biomech 2006; 39 (2): 246-54.

[39] Alkner BA, Tesch PA, Berg HE. Quadriceps EMG/force relationship in knee extension and leg press. Med Sci Sports Exerc 2000; 32(2): 459-63.

[40] Ono T, Yagi R, Ihashi K, et al. The influence of knee rotation on electromyographic activity of medial and lateral heads of the quadriceps femoris muscle during isometric knee extension effort. J Phys Ther Sci 2002; 14 (1): 57-62.

[41] Cuddeford T, Williams AK, Medeiros JM. Electromyographic activity of the vastus medialis oblique and vastus lateralis muscles during selected exercises. J Man Manip Ther 1996; 4(1): 10-5.

[42] Cerny K. Vastus medialis oblique/vastus lateralis muscle activity ratios for selected exercises in persons with and without patellofemoral pain syndrome. Phys Ther 1995; 75(8): 26-38.

[43] Pincivero DM, Coelho AJ, Campy RM, Salfetnikov Y, Suter E. Knee extensor torque and quadriceps femoris EMG during perceptually-guided isometric contractions. J Electromyogr Kinesiol 2003; 13 (2): 159-67.

(C) Kushion et al.; Licensee Bentham Open.

This is an open access article licensed under the terms of the Creative Commons Attribution Non-Commercial License (http: //creativecommons.org/licenses/by$\mathrm{nc} / 3.0 /$ ) which permits unrestricted, non-commercial use, distribution and reproduction in any medium, provided the work is properly cited. 